\title{
BLOOD GLUCOSE LEVEL MONITORING BY NONINVASIVE METHOD USING NEAR INFRA RED SENSOR
}

\author{
P.Daarani ${ }^{1} \&$ A.Kavithamani ${ }^{2}$
}

\begin{abstract}
Diabetes is a common chronic disease in mostly all countries worldwide. The most commonly used method to measure glucose level in blood is an invasive method which is painful, expensive and danger in spreading infectious diseases. Over a long term, the invasive method results in damage of finger tissues. As an alternative, the noninvasive method can be used which facilitates frequent testing, relieves pain and discomfort caused by frequent finger pricks. A noninvasive method of glucose level measurement is proposed in this paper. The variation in the intensity of NIR light received from the photo detector after passing through the finger is used to determine the glucose level of blood. The measured glucose level is displayed in LCD display and also transmitted to the android application which is created in the mobile phone to display and store data via Bluetooth.

Keywords - Glucose measurement, Noninvasive method, NIR, Android application, Bluetooth
\end{abstract}

\section{INTRODUCTION}

Monitoring of glucose level of blood is important to avoid complications of diabetic and damage to organs. Since invasive method of glucose level measurement is painful and causes damage to nerves, non-invasive method is used as an alternative.

Shinde and Prasad [1] described a noninvasive glucose monitoring method in which the NIR is sent through the fingertip and over-systolic pressure is applied to the finger to stop the flow of blood for a period of 30 seconds. The response of the optical signal thus obtained is studied by performing the FFT analysis using the spectrum analyzer.

Chi Fuk So at.el [2] reviewed recent advances in noninvasive glucose monitoring and concluded that optical method is one of the painless and promising methods that can be used for noninvasive blood glucose measurement. Jyoti Yadav [3] used NIR LED of 940nm wavelength to analyze the glucose concentration by conducting experiment on the human forearm.

The noninvasive blood glucometer design containing two LEDs of the same wavelength with one acting as photo emitter and the other as the photodetector is proposed in [4]. In [5] a total of 8 LED pairs were tested for sensitivity to different glucose concentration and it is reported that among all the LED pairs tested, the most effective pair was NIR LED with the wavelength of $1450 \mathrm{~nm}$. Three different probes (arm, finger, ear lobe) were designed to measure blood glucose using $940 \mathrm{~nm}$ NIR LED. Parag et.al [6] placed emitter and detector on the same side of the finger to detect the reflected signals properly, hence a phase shift of $180^{\circ}$ occurs between transmitted and reflected signals.

This paper presents a design and development of noninvasive blood glucose measurement sensor system which consists of NIR light source of $940 \mathrm{~nm}$ wavelength. By measuring the intensity of the light received after passing through the finger, blood glucose level can be calculated. An android application (app) is created to display and store the measured glucose value along with date and time in a text file which can be viewed at any time. The main aim of this work is to develop a simple, reliable, painless, cost effective and portable device for glucose measurement.

The organization of the paper is as follows. In section II principle of blood glucose measurement has been discussed. Section III discusses the block diagram of proposed work and system design is detailed in section IV. An illustration is provided in section $\mathrm{V}$ and section VI concludes the paper.

\section{PRINCIPLE OF BLOOD GLUCOSE MEASUREMENT}

When a light ray passes through biological tissues, it is both absorbed and scattered by the tissues. Light scattering occurs in biological tissues due to the mismatch between the refraction index of extracellular fluid and the membranes of the cells. Variation in glucose level in blood affects the intensity of light scattered from the tissue. Beer-Lambert Law plays a major role in absorbance measurement which states that absorbance of light through any solution is in proportion with the concentration of the solution and the length path traveled by the light ray [8]. Light transport theory describes light attenuation as $\mathrm{I}=\mathrm{I}_{0} \mathrm{e}^{-\mu} \mathrm{eff}^{\mathrm{L}}$

where, $\mathrm{I}$ is the reflected light intensity, $\mathrm{I}_{0}$ is the incident light intensity and $\mathrm{L}$ is the optical path length inside the tissue. Attenuation of light inside the tissue depends on the coefficient known as effective attenuation coefficient ( $\left.\mu_{\text {eff }}\right)$, which is given by

\footnotetext{
${ }^{1}$ Department of Electrical and Electronics Engineering, Coimbatore Institute of Technology, Coimbatore, Tamilnadu, India
}

${ }^{2}$ Department of Electrical and Electronics Engineering, Coimbatore Institute of Technology, Coimbatore, Tamilnadu, India 
$\mu_{\text {eff }}=\left[3 \mu_{\mathrm{s}}\left(\mu_{\mathrm{s}}+\mu_{\mathrm{s}}\right)\right]^{1 / 2}$

The absorption coefficient $\left(\mu_{\mathrm{a}}\right)$ is defined as the probability of absorption of photons inside the tissue per unit path length, which is given by

$\mu_{\mathrm{a}}=2.303 \in \mathrm{C}$

$\in$ is the molar extinction coefficient, $\mathrm{C}$ is the tissue chromophore concentration and the reduced scattering coefficient $\left(\mu_{\mathrm{s}}^{\prime}\right)$ is given by equation 4 .

$\mu_{\mathrm{s}}^{\prime}=\mu_{\mathrm{s}}(1-\mathrm{g})$

where g is anisotropy and $\mu_{\mathrm{s}}$ is scattering coefficient. Hence from the equations (1) to (4) it can be concluded that $\mu_{\mathrm{a}}$ depends on the glucose concentration in blood. Thus with the increase in blood glucose concentration, the scattering property of blood decreases.

\section{BLOCK DIAGRAM OF PROPOSED WORK}

The proposed work is based on NIR optical technique. NIR light source of $940 \mathrm{~nm}$ wavelength is chosen because it is suitable for measuring blood glucose concentration. The sensing unit consists of NIR emitter and NIR receiver (photodetector) positioned on either side of the measurement site (fingertip) as shown in figure1. When the NIR light is propagated through the fingertip in which it interacts with the glucose molecule, a part of NIR light gets absorbed depending on the glucose concentration of blood and remaining part is passed through the finger tip. The amount of NIR light passing through the fingertip depends on the amount of blood glucose concentration.

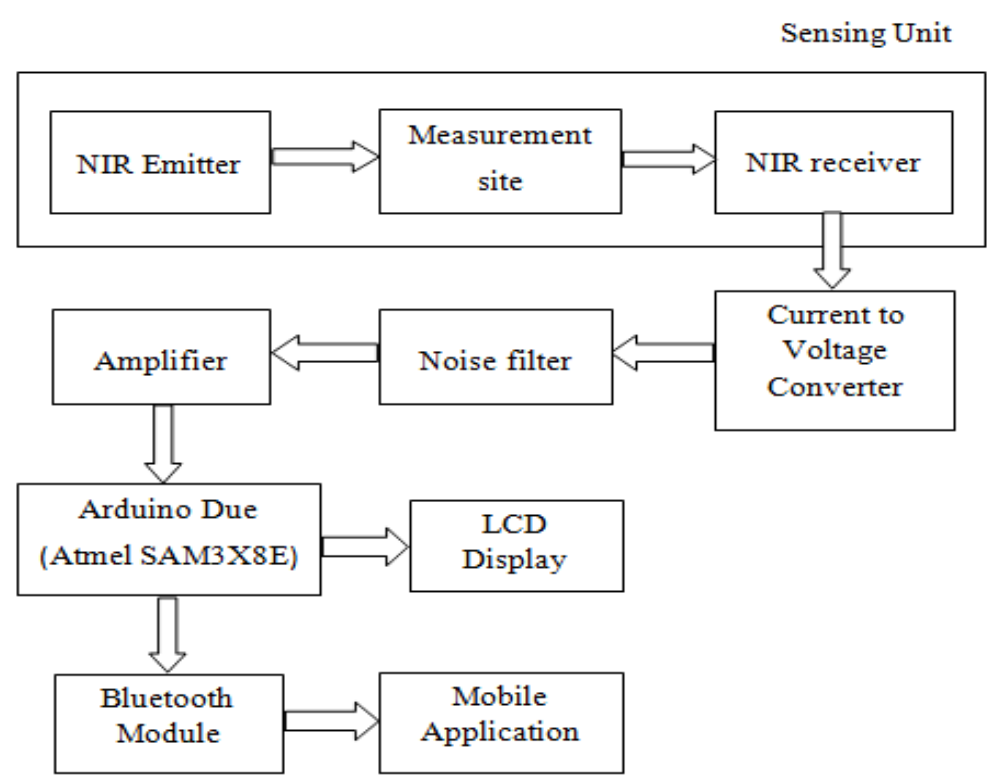

Figure 1. Block diagram of noninvasive glucose concentration measurement system

The transmitted signal is detected by the photodetector. The output current of the photo detector is converted into voltage signal and then it is filtered and amplified. This amplified signal is fed into Atmel SAM3X8E microcontroller. The inbuilt $\mathrm{ADC}$ block is used for converting the received analog signal to digital form. This digital signal is processed by using second order regression analysis to predict the blood glucose value and the blood glucose value is displayed on the LCD display. A mobile application (App) is created in order to view and store the predicted blood glucose value after receiving it via Bluetooth. Atmel communicates to the mobile app via Bluetooth by connecting a Bluetooth module (HC-05) to it. The flow chart of proposed work is shown in figure 2 . 


\section{SYSTEM DESIGN}

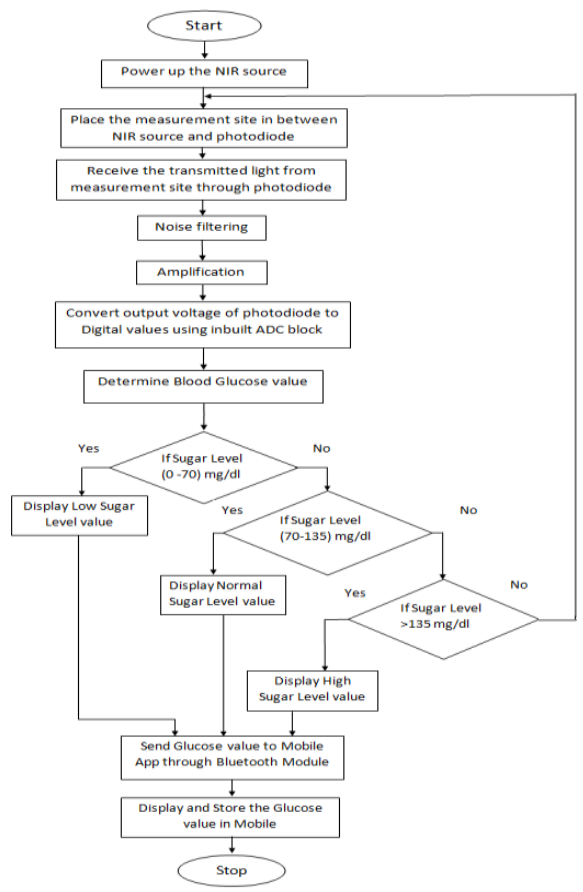

Figure 2. Flow chart of proposed work

The circuit diagram of the designed system consists of filtering stage and amplification stage as shown in figure 3 . The electrical current obtained from the photo detector is converted into the voltage by placing the load resistance $\mathrm{R}_{4}=50 \mathrm{k} \Omega$ at the anode side of photodiode. The cut-off frequency of high pass filter and low pass filter are designed as $2.34 \mathrm{~Hz}$ and 1.59 $\mathrm{kHz}$ respectively.

Cut off frequency of LPF $=1 /\left(2 \pi \mathrm{R}_{1} \mathrm{C}_{1}\right)=1 /\left[2 \pi\left(1 * 10^{3}\right)\left(100^{*} 10^{-9}\right)\right]=1.59 \mathrm{kHz}$

Cut off frequency of HPF $=1 /\left(2 \pi \mathrm{R}_{2} \mathrm{C}_{2}\right)=1 /\left[2 \pi\left(68^{*} 10^{3}\right)\left(1 * 10^{-6}\right)\right]=2.34 \mathrm{~Hz}$

Voltage gain $=1+\left(\mathrm{R}_{\mathrm{f}} / \mathrm{R}_{\mathrm{in}}\right)=1+\left[\left(680^{*} 10^{3}\right) /\left(68 * 10^{3}\right)\right]=101$

The amplified output voltage is connected to analog pin $\mathrm{A}_{0}$ of Arduino due microcontroller for converting the analog signal into digital values. This digital value corresponds to the glucose level. From this digital value, the actual glucose level is determined using polynomial regression equation. This equation is formed from the glucose levels obtained from the laboratory using invasive measurement.

A mobile app is created for displaying and storing the predicted glucose value. Bluetooth module (HC-05) is connected to Arduino due microcontroller in order to communicate with the mobile app via Bluetooth.
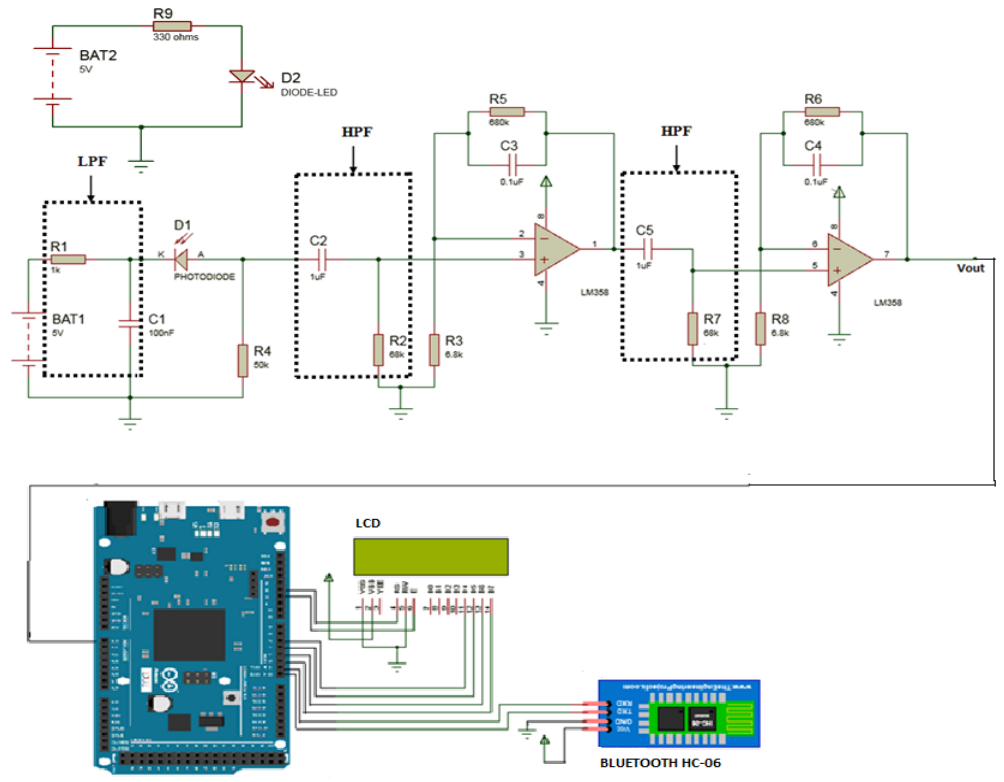

Figure 3. Circuit diagram of designed system 
Once the mobile app is connected to the microcontroller via bluetooth, the glucose value will be displayed in the mobile app screen. The date and time during glucose measurement process will also be stored along with received data using date picker and time picker option in the mobile app. The stored glucose data can also be viewed in the mobile app at any time when restore button is clicked.

\section{ILLUSTRATION}

To obtain polynomial regression equation, 24 diabetic individuals including both genders are considered. Glucose level of these individuals is measured in the laboratory by the invasive method and at the same time the analog voltage corresponding to their glucose level is also measured using the proposed hardware setup as shown in figure 4 and the readings are tabulated in table1.

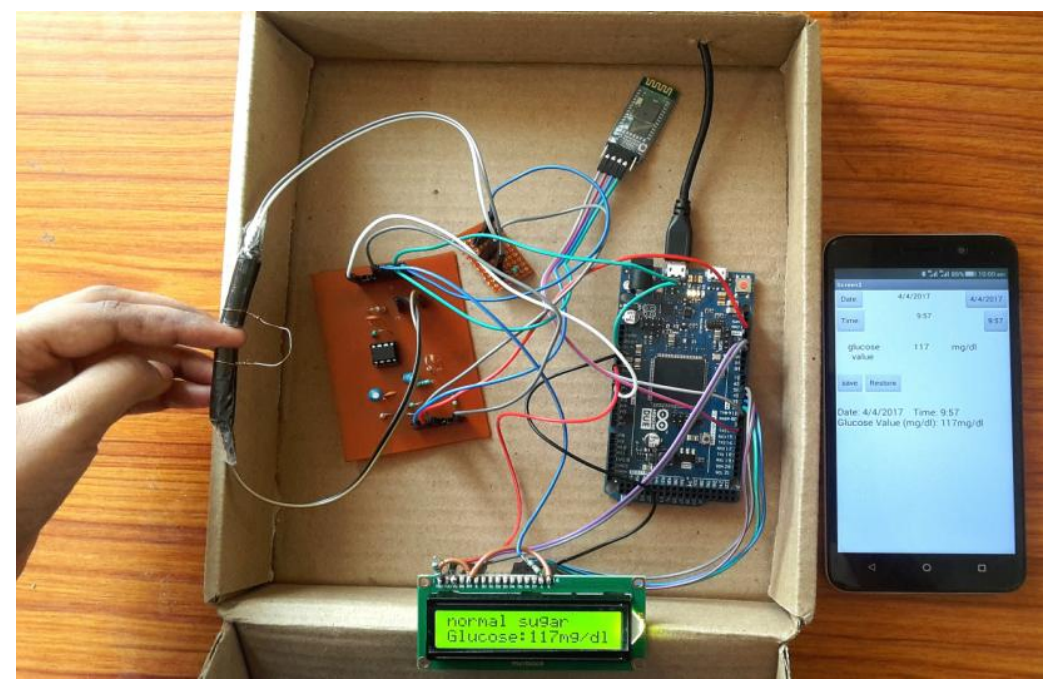

Figure 4. Hardware setup

Table -1 Analog Voltage and the Glucose Level of Samples

\begin{tabular}{|l|l|l|l|l|l|}
\hline S.No & Analog Voltage $(\mathrm{mV})$ & Glucose Level $(\mathrm{mg} / \mathrm{dl})$ & S.No & Analog Voltage $(\mathrm{mV})$ & Glucose Level $(\mathrm{mg} / \mathrm{dl})$ \\
\hline 1 & 499 & 142 & 13 & 607 & 196 \\
\hline 2 & 509 & 146 & 14 & 627 & 191 \\
\hline 3 & 519 & 156 & 15 & 695 & 167 \\
\hline 4 & 519 & 157 & 16 & 735 & 220 \\
\hline 5 & 548 & 177 & 17 & 612 & 244 \\
\hline 6 & 524 & 159 & 18 & 847 & 247 \\
\hline 7 & 543 & 209 & 19 & 833 & 248 \\
\hline 8 & 568 & 133 & 20 & 867 & 276 \\
\hline 9 & 573 & 179 & 21 & 935 & 302 \\
\hline 10 & 583 & 22 & 999 & 321 \\
\hline 11 & 592 & 175 & 23 & 1136 & 338 \\
\hline 12 & 597 & 187 & 24 & 1538 & 516 \\
\hline
\end{tabular}

The analog voltage measured at analog pin $\mathrm{A}_{0}$ of Arduino due microcontroller and the corresponding glucose concentration measured by the invasive method in the laboratory are plotted on a graph and shown in figure 5. The polynomial equation relating the analog voltage and the glucose level is computed by using regression tool and shown below.

$y=\left(8 * 10^{-5}\right) x^{2}+0.1873 x+46.131$

where $\mathrm{x}$ and $\mathrm{y}$ are analog voltage $(\mathrm{mV})$ and glucose level $(\mathrm{mg} / \mathrm{dl})$ respectively. 


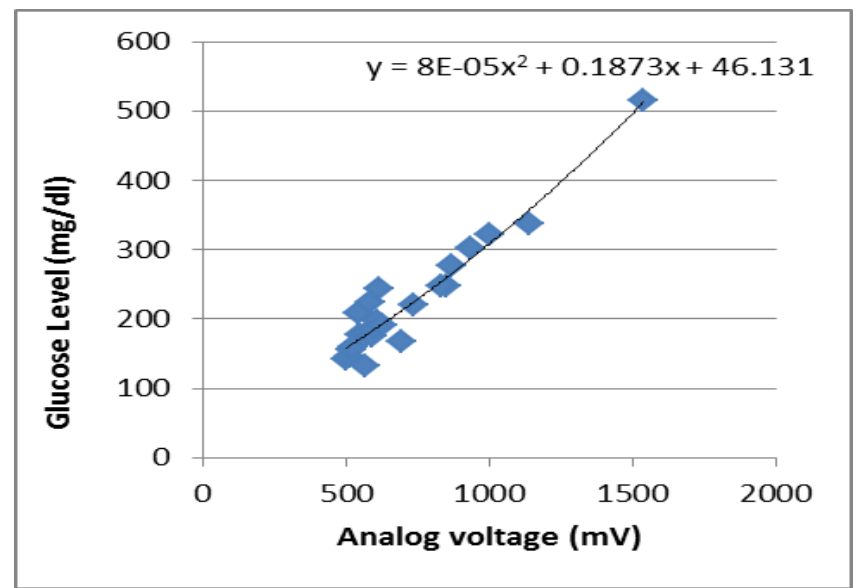

Figure 5. Regression analysis of glucose data with analog voltage data

Arduino program is written to determine the glucose level for the given analog voltage using (5). The continuous analog voltage values received from the photo detector while placing the finger in-between NIR emitter and photo detector are stored in an array and they are averaged. The microcontroller calculates the glucose value corresponding to this average analog voltage using (5) and displays it on both LCD display and mobile app. The calculated glucose value is also stored in the mobile along with date and time and it can be viewed at any time.

\section{VALIDATION:}

The proposed method is validated by measuring the glucose reading of 40 individuals using both invasive and proposed noninvasive method and the readings are tabulated in table 2 . The accuracy of the proposed glucose measurement device is measured using Clarke Error Grid Analysis and Surveillance Error grid analysis.

Table - 2 Comparison of Results

\begin{tabular}{|l|l|l|l|l|l|l|l|}
\hline S.No & $\begin{array}{l}\text { Glucose Value } \\
\text { Obtained by } \\
\text { Invasive method }\end{array}$ & $\begin{array}{l}\text { Glucose Value } \\
\text { Obtained by Non } \\
\text { Invasive method }\end{array}$ & Difference & S.No & $\begin{array}{l}\text { Glucose Value } \\
\text { Obtained by } \\
\text { Invasive method }\end{array}$ & $\begin{array}{l}\text { Glucose Value } \\
\text { Obtained by Non } \\
\text { Invasive method }\end{array}$ & Difference \\
\hline 1 & 117 & 118 & +1 & 21 & 145 & 156 & -11 \\
\hline 2 & 143 & 143 & 0 & 22 & 170 & 161 & 9 \\
\hline 3 & 112 & 115 & +3 & 23 & 113 & 108 & 5 \\
\hline 4 & 106 & 103 & -3 & 24 & 110 & 152 & -42 \\
\hline 5 & 166 & 169 & +3 & 25 & 165 & 152 & 13 \\
\hline 6 & 193 & 192 & -1 & 26 & 227 & 258 & -31 \\
\hline 7 & 88 & 88 & 0 & 27 & 149 & 160 & -11 \\
\hline 8 & 108 & 110 & +2 & 28 & 130 & 104 & 26 \\
\hline 9 & 110 & 117 & -7 & 29 & 220 & 198 & 22 \\
\hline 10 & 134 & 151 & -17 & 30 & 129 & 136 & -7 \\
\hline 11 & 245 & 213 & 32 & 31 & 316 & 268 & 48 \\
\hline 12 & 299 & 252 & 47 & 32 & 131 & 128 & 3 \\
\hline 13 & 145 & 139 & 6 & 33 & 192 & 220 & -28 \\
\hline 14 & 211 & 186 & 25 & 34 & 148 & 157 & -9 \\
\hline 15 & 152 & 111 & 41 & 35 & 170 & 162 & 8 \\
\hline 16 & 205 & 219 & -14 & 36 & 145 & 154 & -9 \\
\hline 17 & 120 & 129 & -9 & 37 & 195 & 181 & 14 \\
\hline 18 & 157 & 142 & 15 & 38 & 92 & 85 & 7 \\
\hline 19 & 117 & 122 & -5 & 39 & 250 & 235 & 15 \\
\hline 20 & 164 & 170 & -6 & 40 & 100 & 108 & -8 \\
\hline & & & & & & \\
\hline
\end{tabular}


(i) The Clarke error grid analysis is used for analyzing accuracy in commercial glucose monitoring devices which are used in self monitoring of glucose. The Clarke error grid analysis is done using MATLAB software where the reference glucose value and predicted glucose value from table 2 are given as input data and the result obtained shows that $95 \%$ of the predicted glucose values are under region A which is clinically acceptable region for glucose measurement devices figure 6 .

\begin{tabular}{|c|c|c|c|c|}
\hline \multirow{4}{*}{ 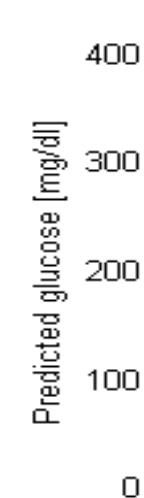 } & \multicolumn{2}{|l|}{ Clarke's Error Grid Analysis } & Region A & $\begin{array}{l}\text { values within } 20 \% \text { of the reference } \\
\text { value }\end{array}$ \\
\hline & & & Region B & $\begin{array}{l}\text { values are outside of } 20 \% \text { but would } \\
\text { not lead to inappropriate treatment. }\end{array}$ \\
\hline & D & D & Region C & $\begin{array}{l}\text { values leading to unnecessary } \\
\text { treatment. }\end{array}$ \\
\hline & 100 & 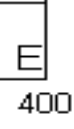 & Region D & $\begin{array}{l}\text { values indicating a potentially } \\
\text { dangerous failure to detect } \\
\text { hypoglycemia or hyperglycemia. }\end{array}$ \\
\hline Figure 6. & $\begin{array}{l}\text { Reference glucose [mg/dl] } \\
\text { Result of Clarke analysis }\end{array}$ & & Region E & $\begin{array}{l}\text { values that would confuse treatment of } \\
\text { hypoglycemia for hyperglycemia and } \\
\text { vice versa. }\end{array}$ \\
\hline
\end{tabular}

(ii) Surveillance Error grid analysis:

The surveillance error grid analysis is mainly used to determine the clinical accuracy and risk range of continuous glucose monitoring device used in intensive care unit. The surveillance error grid analysis of readings of table 2 is shown in figure 7 . It shows that most of the glucose readings are in dark green zone (no risk) and few glucose readings are in light green zone (slight risk). Hence the proposed method is acceptable and suitable for monitoring glucose concentration in blood.

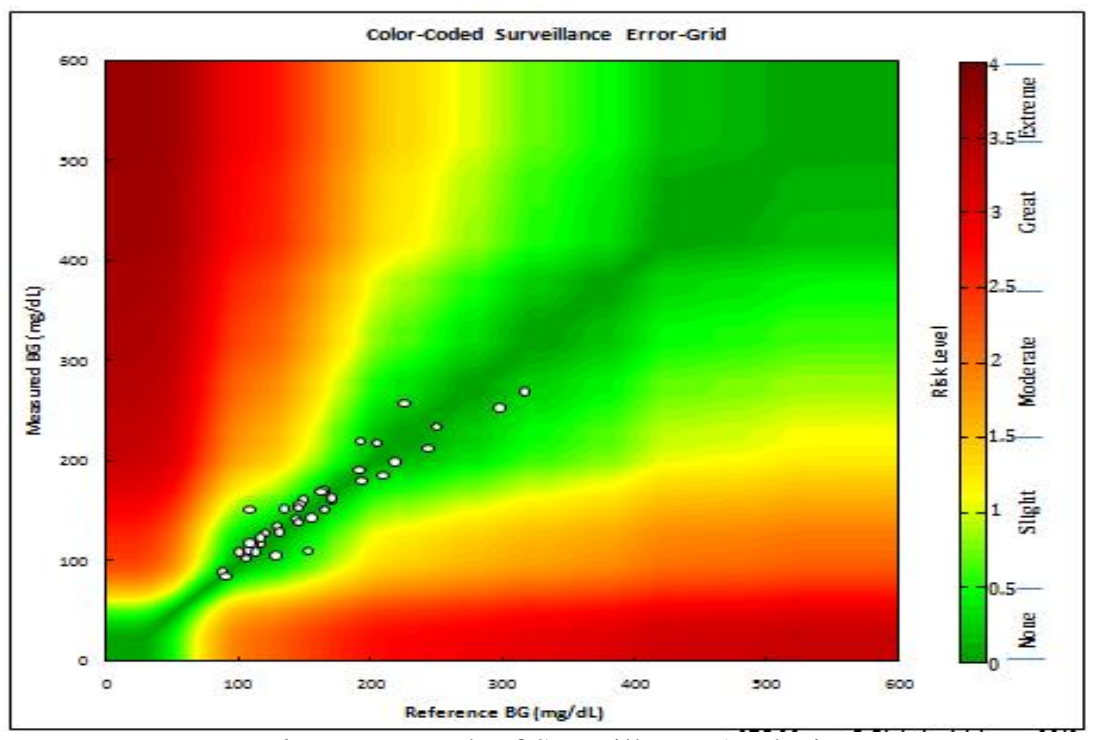

Figure 7. Result of Surveillance Analysis

\section{CONCLUSION}

Invasive method of glucose measurement is painful, costly and discomfort. It also has a risk of infection and not used for continuous monitoring. In order to overcome the above disadvantages, a noninvasive method for blood glucose measurement using near-infrared LED is proposed in this paper. The glucose level in the blood which is obtained from the photodetector is displayed in both the LCD display and the developed mobile app. The proposed method is validated using error grid analyses. This portable noninvasive blood glucose monitor provides a very effective means for assisting the health care management of diabetic patients. This can be used for monitoring blood glucose level of the patients in the home as well as health care centers. 


\section{REFERENCES}

[1] Prof.Mrs.A.A.Shinde, "Non Invasive Blood Glucose Measurement using NIR technique based on occlusion spectroscopy", International Journal of Engineering Science and Technology (IJEST) ISSN: 0975-5462 Vol. 3 No. 12, pp. 8325-8333, December 2011.

[2] Chi-Fuk So, Kup-Sze Choi, Thomas KS Wong, Joanne WY Chung, "Recent advances in non invasive glucose monitoring", Medical Devices: Evidence and Research. Vol.5, pp. 45-52, June 2012.

[3] JyotiYadav, Asha Rani, Vijender Singh "Near-Infrared LED based Non-invasive Blood Glucose Sensor", International conference on Signal Processing and Integrated Network(SPIN), IEEE, pp. 591-594, Feb 2014.

[4] Christopher Dale Chua, Ian Mikhael Gonzales, Enrique Manzano, Maria Carla Manzano, "Design and Fabrication of a Non-Invasive Blood Glucometer Using Paired Photo-Emitter and Detector Near-Infrared LEDs”, DLSU Research Congress, De La Salle University, Manila, Philippines, March 2014

[5] JyotiYadav, Asha Rani, Viajnder Singh, BhaskarMoahnMurari, "Comparative Study of Different Measurement Sites using NIR Based Non-invasive Glucose Measurement system”, Procedia Computer Science, Elsevier, Vol. 70, pp. 469 - 475, 2015.

[6] Parag Narkhede, Suraj Dhalwar, B. Karthikeyan. "NIR Based Non-Invasive Blood Glucose Measurement", Indian Journal of Science and Technology, Vol. 9 No. 41, pp. 1-7, November 2016.

[7] Maier JS, Walker SA, Fantini S, Franceschini MA, Gratton E., "Possible correlation between blood glucose concentra-tion and the reduced scattering coefficient of tissues in the near infrared", Optics letters. Vol. 19 No. 41, pp. 2062-64, December 1994. 